\title{
Überregionale Dimensionen der Literatur aus der Mährischen Walachei \\ Facetten der Moderne im Schaffen Heinrich Herbatscheks
}

\author{
Libor Marek
}

The paper presents an analysis of the prose volume Sketchbook by the writer Heinrich Herbatschek (1877-1956). This Austrian author came from Moravian Wallachia (Vsetín) and in his works he provides evidence for a specific reception of the aesthetic modernism by marginal and regional authors. In Sketchbook (1929), a late modernist text, Herbatschek uses an impressionist and decadent form of language. The modernist arrangement and traditional, humanist and enlightened ideals form an ambivalent symbiosis in this text.

Heinrich Herbatschek - Moravian Wallachia - Moravian German literature - literary modernism - decadence - impressionism

Der vorliegende Artikel bietet eine Analyse des Prosabandes Skizzenbuch (1929) von Heinrich Herbatschek (1877-1956). Dieser aus dem mährisch-walachischen Ort Wsetin (Vsetín) stammende österreichische Schriftsteller liefert in seinem Werk Beweise für eine spezifische Rezeption der ästhetischen Moderne seitens marginaler und regionaler Autoren. In dem Skizzenbuch, einem spätmodernistischen Text, bedient sich Herbatschek einer impressionistischen und dekadenten Formensprache. Das modernistische Arrangement geht hier eine ambivalente Symbiose mit traditionellen, humanistisch-aufklärerischen Wertvorstellungen ein.

Heinrich Herbatschek - Mährische Walachei - deutschmährische Literatur - literarische Moderne - Décadence - Impressionismus.

\section{Mährische Walachei mit und ohne Grenzen}

Der nachfolgende Artikel $^{1}$ berührt einen partiellen Aspekt jenes vielschichtigen literarischen Phänomens, das insbesondere im Kontext der ästhetischen Moderne zu den weitgehend unerforschten Kapiteln der deutschen bzw. österreichischen Literaturgeschichte gehört, und zwar die deutsche Literatur aus der Mährischen Walachei. Dieses tschechische Gebiet brachte, trotz seiner relativ dünnen deutschen Besiedlung, ${ }^{2}$ eine Literatur hervor, die als spezifischer, jedoch integraler Bestandteil der deutschmährischen Literatur anzusehen ist. Im Allgemeinen kann man von Krappmanns (2013:10) Diktum bezüglich der deutschmährischen Autoren ausgehen und analog behaupten: Ein mährisch-walachischer Autor ist jener, welcher

1 Dieser Beitrag entstand im Rahmen des Forschungsprojekts Deutsche Literatur und Kultur in der Mährischen Walachei: Die europäische Dimension eines regionalen kulturellen Diskurses, das von der Grantová agentura České republiky gefördert wird (GA CR, Reg. Nr. 16-11983S).

2 Bspw. in Bistritz am Hostein lag im Jahr 1880 der Anteil der Deutschen an der Gesamtbevölkerung bei 6,2 \%, der Anteil der meistens deutschsprachigen Juden fiel noch geringer aus: 2,6\% (vgl. Bartoš, Schulz und Trapl 1980: 162). 
sein ganzes oder einen Teil seines Lebens auf dem Gebiet der Mährischen Walachei verbracht hat.

In diesem Zusammenhang scheint es notwendig, ein geeignetes komplexes Raummodell für die kultur- und literaturhistorische Erfassung dieser Region einzuführen, wobei sich logischerweise - wie im Fall anderer mährischer Gebiete - die Kategorie der Grenze als konstitutives Merkmal für eine solche Erfassung anbietet. Bereits bei grobem Hinsehen versagt jedoch die Grenze als Erfassungsmodus, denn man stößt eher auf eine Reihe von Zwischenräumen und Interaktionen zwischen heterogenen Entitäten in der Mährischen Walachei. Die Walachei stellt nämlich laut Brouček und Jeřábek (2007:1101) ein historisch-ethnografisches Gebiet im östlichen Teil Mährens mit unüberschaubaren Grenzziehungen dar. Sie war nie eine selbständige Verwaltungseinheit und zeichnet sich durch eine strittige geografische Abgrenzung und einen deutlichen Konstruktcharakter mit diversen Differenzierungsmerkmalen bezüglich der Sprache, der Religion und vor allem der Ethnizität aus. Insbesondere in der zweiten Hälfte des 19. Jahrhunderts und im beginnenden 20. Jahrhundert war die Walachei ein multikultureller Raum, in dem neben Tschechen auch Deutsche und Juden lebten und kulturelle Spuren hinterließen, wobei die Deutschen oft keine Deutschen, sondern als Deutsche getarnte Tschechen oder deutschsprachige Juden waren. Das Deutschtum funktionierte als Zeichen der Zugehörigkeit zu gehobenen gesellschaftlichen oder kulturellen Kreisen. Die Träger der deutschen Kultur waren traditionell die deutsche bzw. deutsch-jüdische Intelligenz und der deutsche Adel. Im Zuge der Industrialisierung der Region in der zweiten Hälfte des 19. Jahrhunderts kamen noch jene Kulturschaffenden hinzu, die sich aus dem Umkreis der reichen mährisch-walachischen Industriellen rekrutierten.

Aus den obigen Ausführungen wird deutlich, dass die deutsche mährischwalachische Literatur nur schwer durch Abgrenzungen (seien es geografische, ethnische oder nationale) kontextualisierbar ist. Von daher kann die Erörterung eines derartigen Phänomens unter Zuhilfenahme der analytischen Kategorie der Grenze leicht in eine Sackgasse geraten. Gefragt ist deswegen ein offenes und dabei umfassendes Konzept. Manfred Weinberg (2014) postuliert in Bezug auf die Kulturräume mit interkulturellem bzw. transkulturellem Charakter das Raummodell des Horizonts als komplementäres, oder eher konkurrierendes Raummodell. Im Unterschied zur Grenze ermöglicht der Horizont die Auslotung von Zwischenräumen, zeitweise gültigen Grenzen und Vermischungen, er generiert ein immenses Interaktionspotential (vgl. Weinberg 2014: 22). Dies scheint ein für die Erforschung der Literatur aus der Mährischen Walachei denkbarer Ausweg zu sein. 


\section{Der Modellfall Herbatschek}

$\mathrm{Zu}$ den mährisch-walachischen Autoren kann auch Heinrich Herbatschek (1877, Wsetin - 1956, Wien) gezählt werden, dessen Werk Skizzenbuch (1929) der untenstenden Analyse zugrunde gelegt wurde. Prägendes Element seines Schaffens sind ebenfalls die Grenzgänge, oder besser gesagt Horizontverschmelzungen im spannungsgeladenen Verhältnis von Zentralität und Marginalität, Originalität und Epigonalität, Deutschtum bzw. Österreichertum und Tschechentum.

Wichtige Momente der Biografie und der literarischen Karriere Herbatscheks erinnern in ihren Grundzügen an die Schicksale anderer aus dieser mährischen Provinz stammenden Autoren: ${ }^{1}$ in der Walachei verbrachte Kindheit und/oder Jugend, Studium in Wien, Karriere in einem von der Literatur weit entfernten Bereich (Jura oder Philosophie), wiederholte Versuche, in der (Wiener) Literaturszene Fuß zu fassen, Nachahmung moderner literarischer Trends, mangelhafte, beinahe ausbleibende Rezeption durch die Zeitgenossen, letztendlich literarische Vergessenheit. Die Unterschiede zwischen den einzelnen Vertretern bestehen dann nicht nur in der thematischen Ausrichtung oder in der Verwendung anderer formaler Gestaltungsprinzipien bei der Abfassung ihrer Werke, sondern auch im Ausmaß der Offenheit gegenüber der entstehenden modernen Welt und in der Reflexion ihrer Entstehung. Unter diesem Gesichtspunkt betrachtet können auch die Hervorbringungen marginaler Autoren, die das aus der Mährischen Walachei stammende Schrifttum repräsentierten, interessante, für die Erforschung der kanonisierten deutschen bzw. österreichischen Literatur relevante Impulse liefern. Dies ist die grundlegende Prämisse der nachfolgenden Textanalyse.

Es geht also unter anderem darum, zu zeigen, dass das Stigma der Marginalität und Regionalität, das dem Schaffen eines vergessenen, aus der mährischwalachischen Provinz stammenden Autors anhaftet, paradoxerweise zur Sprengung des regionalen Rahmens und zur Thematisierung überregionaler Probleme mit überzeitlicher Relevanz führen kann. Anders gesagt: Die Wahrnehmung der Grenze muss durch die Wahrnehmung des Horizonts ersetzt werden. Aus dieser Perspektive betrachtet ist die regionale Dimension der Literatur nicht zu unterschätzen und sie sollte nicht übersehen werden. Dies betont auch Jörg Krappmann (2012: 23 ff.) in seinem Ansatz, wobei er insbesondere auf die Funktion der Regionalität im Prozess der Rezeption der ästhetischen Moderne seitens der regionalen, später in den großen Zentren (etwa in Wien) lebenden Autoren hinweist, deren Werke laut Krappmann eine Art Brücke von der nicht kanonisierten zur hohen Literatur darstellen.

1 Beispielsweise die aus Bistritz am Hostein stammende Philosophin und Schriftstellerin Susanne Schmida-Wöllersdorfer (1894-1981), deren vor Kurzem entdeckter, dokumentierter und zum Teil erforschter literarischer Nachlass - überwiegend expressionistische Dramen, Lyrik, Essays und esoterische Texte - ein Schattendasein in einem Wiener Haus führte. 
Auch unter Berücksichtigung dieses Umstandes öffnet die vorliegende Studie einen ersten, aber notwendigerweise eingeengten Blick auf das poetische Schaffen Heinrich Herbatscheks und sie ist daher als erster Ansatz zur literaturhistorischen Kontextualisierung der Werke dieses wenig bekannten Autors aufzufassen. Heinrich Herbatschek gehört nämlich zu denjenigen Schriftstellern, deren Schaffen für die literarische Historiografie immer noch eine Terra incognita darstellt ${ }^{1}$ und dessen Name äußerst spärlich in Autorenlexika aufzufinden ist. ${ }^{2}$ In der einschlägigen Forschungsliteratur figuriert Herbatschek höchstens unter marginalen Schriftstellern, die die tschechische Literatur dem deutschsprachigen Rezipientenkreis näher brachten. Seine eigenen Texte wurden zwar noch zu Herbatscheks Lebzeiten herausgegeben, jedoch kaum rezipiert. Das liegt zum Teil an ihrem epigonalen Charakter, aber auch an der verblüffenden Vielfalt und Polyfunktionalität seines sperrigen Euvres, das zwischen juristischen Kommentaren und fiktionalen Texten oszilliert.

Heinrich Herbatschek stammte aus einer bürgerlichen jüdischen Familie, sein Vater Friedmann war Prokurist. Der gebürtige Wsetiner besuchte das Piaristengymnasium in der mährischen Stadt Kremsier und anschließend studierte er Jura an der Prager und Wiener Universität. Nach der Promotion in Wien im Jahr 1901 wirkte er als Rechtsanwalt, seit 1919 sogar als Funktionär der österreichischen Rechtsanwaltskammer. Im Laufe des Ersten Weltkriegs engagierte er sich zugunsten verwundeter und kranker Soldaten. In der Kaserne unterrichtete Herbatschek Tschechisch, was schließlich von der Staatsmacht als subversive Tätigkeit und gefährliche ideologische Beeinflussung österreichischer Soldaten interpretiert wurde. Wegen seiner Kontakte zu den Repräsentanten des tschechischen öffentlichen Lebens wurde er von der Polizei überwacht, persekuiert und in ein Archiv abgeschoben. Auch seine Vortragstätigkeit im Wiener Volksbildungshaus Urania bot insbesondere wegen seiner pazifistischen Rhetorik Anlässe zur strafrechtlichen Verfolgung, die aber zu keiner Verurteilung führte.

Heinrich Herbatschek lebte in einem äußerst turbulenten Zeitalter und war Zeuge eines brisanten nationalen, sozialen und politischen Umbruchs im Verlauf der letzten Dezennien der Existenz der Habsburgermonarchie. Industrialisierung, Geldherrschaft, Pragmatismus, Nationalismus prägten das gesellschaftliche Leben in der zweiten Hälfte des 19. Jahrhunderts in Österreich-Ungarn. Nach dem Ersten Weltkrieg zerfiel der Vielvölkerstaat unwiederbringlich und es entstanden die Nachfolgestaaten, darunter die Tschechoslowakei, die für Herbatschek auch

1 Eine Pionierleistung im Bereich der Biografie und Herausgebertätigkeit Herbatscheks leistete die an der Masaryk-Universität in Brünn 2009 verteidigte Magisterarbeit Heinrich Herbatschek als Herausgeber der Zeitschrift „Der Nachbar" von Tomáš Hájek. Es handelt sich um eine materialreiche Quellenbasis, auf die hier bezüglich der grundsätzlichen Lebensfakten zurückgegriffen werden konnte. Einige Angaben bedürfen allerdings Ergänzungen und Korrekturen.

2 Bspw. Handbuch österreichischer Autorinnen und Autoren jüdischer Herkunft. 18. bis 20. Jahrhundert. (2002: 530). 
weiterhin einen relevanten kulturellen Bezugspunkt darstellte. Diesbezüglich passen Herbatscheks Lebensweg und -haltungen gar nicht ins Bild eines aus Mähren stammenden, konservativ gesinnten Autors wie etwa Richard von Schaukals. Vice versa bei Herbatschek, der sich immer auf die humanistische Linie in der tschechischen Geistesgeschichte berief:

\begin{abstract}
„Die czechoslavische Nation, welche sich im neunten Jahrhundert bereits das Christentum zu Eigen gemacht hat, dankt diesem zunächst ihre Kultur, ihr Geistesleben. Und so oft sich ein Zweig des Stammes gegen die religiöse Macht auflehnte, war auch stets ein Aufgang der Kultur geöffnet. Komensky, Huß, Masaryk sind leitende Geistesheroen im Werden der Nation. Kultur und Aufklärung war und ist das ewig junge Humanitätsideal: Kollár, Palacký, Havliček und der Philosoph Smetana seine Prediger.“ (Herbatschek 1913: 5)
\end{abstract}

Herbatschek war also kein verschmähter Ästhet und postkakanischer Monarchist mit modernistischen Allüren, dessen Welt mit dem Zusammenbruch ÖsterreichUngarns zerfallen wäre und der sie mit Verstiegenheit auf geistiger Basis wieder künstlich konstruiert hätte. Er zeigte - wie kein anderer aus der östlichen Ecke Mährens stammender, deutschschreibender Autor - reges Interesse für sein Herkunftsland auch nach dem Zerfall Österreich-Ungarns. Herbatschek verkehrte mit den großen Figuren der tschechischen Kultur und Politik. Rückblickend fällt insbesondere die auf Glorifizierung beruhende Beziehung zum Philosophen und ersten tschechoslowakischen Staatspräsidenten Tomáš Garrigue Masaryk, den er wahrscheinlich schon 1896 während seines Studiums an der Karl-FerdinandUniversität in Prag kennengelernt hatte und dessen Buch Die Ideale der Humanität er 1902 ins Deutsche übertrug, stark ins Gewicht. Gerade dieses Werk und dessen Botschaft kann nun als paradigmatisch angesehen werden, denn nicht nur als Jurist, sondern auch als antinationalistisch, kosmopolitisch und humanistisch gesinnter Literat betonte Herbatschek in seinen Werken das Ethos des Lebens. Auch die regionale Verankerung verband beide Männer, denn Masaryk wirkte als Abgeordneter im österreichischen Reichsrat für den Wahlbezirk Mährische Walachei, in der auch Herbatschek heimisch war. Herbatschek repräsentiert somit eine einzigartige deutsche, auf tschechischem Boden feststellbare Tradition, nämlich die des kulturellen Austausches und der Kooperation mit den Tschechen. Und gerade diese Bemühung markierte auch seine Schritte auf dem Weg von der Regionalität zum europäischen Kulturrahmen.

Bis 1909 publizierte Herbatschek in den Wiener Periodika Neue Freie Presse, Österreichische Wochenschrift, Die Zeit, Der Weg: Wochenschrift für Politik und Kultur Zeitschrift für Handel und Gewerbe und in vielen weiteren. Nach dem Krieg veröffentlichte er seine Artikel in der von der Brünner Kosmopolitischen Gesellschaft herausgegebenen Zeitschrift Die Wahrheit, welche den in den 1920er Jahren grassierenden Nationalismus und Chauvinismus sowohl in der Tschechoslowakei als 
auch in Europa anprangerte. Herbatschek war Vizepräsident des Deutsch-czechischen Komitees (1911-1914), er engagierte sich als Vorsitzender im Verein der Mährer und in der 1928 gegründeten Österreichisch-Tschechoslowakischen Gesellschaft, deren Mitteilungsblatt Der Nachbar er herausgab. Daneben übersetzte Herbatschek die moderne tschechische Literatur, woraus auch seine herausgeberischen Aktivitäten herrührten. Er übertrug einen Teil des Werkes des tschechischen Modernisten und Mitverfassers des Manifests der Tschechischen Moderne, Josef Svatopluk Machars (1864-1942), ins Deutsche.

Bis 1938 lebte Herbatschek in Wien, 1938 zog er wahrscheinlich wegen drohender Nazigefahr nach Prag um und seine Spuren verlieren sich wiederum im Jahr 1940 in Wien. In diesem Zeitraum endet auch Hájeks (2009: 4) Rekonstruktion des Lebensweges von Herbatschek. Aufgrund fehlender oder unvollständiger Quellennachweise schließt sie nicht einmal einen tragischen Ausgang der Lebensgeschichte des Mährischen Walachen aus. Ob seine beabsichtigte Ausreise in die Vereinigten Staaten erfolgte, sei dahingestellt. Überraschenderweise sind aber dem Eintrag zu Heinrich Herbatschek in der genealogischen Datenbank (Geni 2017) das Sterbedatum 30. Juni 1956 und der Sterbeort Wien zu entnehmen. Man kann also davon ausgehen, dass Herbatschek den Zweiten Weltkrieg überlebte und bis zu seinem Tod in Österreich verblieb. Höchstwahrscheinlich gab er dann seine schriftstellerische und publizistische Tätigkeit völlig auf, weil keine nach dem Zweiten Weltkrieg entstandenen Werke von ihm vorliegen.

\section{Vom Recht zum Krieg und wieder zurück}

Die poetischen Werke Herbatscheks sind unter einer dicken Schicht soziologisch oder psychologisch gefärbter rechtswissenschaftlicher ${ }^{1}$ und politischer $^{2}$ Abhandlungen wie auch humanistisch geprägter Reflexionen ${ }^{3}$ versteckt. Man stößt bei ihm sogar auf Reiseliteratur. ${ }^{4}$ Einen nicht zu unterschätzenden Beitrag zum deutsch-tschechischen politischen und kulturellen Dialog stellen hingegen

1 Stempel und Gebühren-Befreiungen (1904), Ausgedinge oder Bauernversicherung (1904), Reformen im österreichischen Verkehrs- und Rechtsleben (1904), Das Gast- und Schankgewerbe im Lichte der Volkswirthschaft der juristischen Praxis (1906), Kinder- und Elternrecht. Volkstümlich dargestellt von Heinrich Herbatschek (1910), Ein Indizienprozess: Der Fall Sautner (1913), Unsere neuen Gesetze. Kleine Sammlung der nach dem Kriege in Geltung bleibenden Kriegsgesetze gemeinverständlich dargestellt und mit Beispielen sowie Vorlagen versehen (1907), Verbrecherinnen: Kriminalpsychologische Studie (ca. 1920), Leitfaden der österreichischen Bürgerkunde: Für den Schulgebrauch (1923), Bürgerkunde zum Gebrauche für Mittelschulen und verwandte Lehranstalten (1926), Gesetzeskunde für Ärzte (1927), Gesetzeskunde für Techniker (1929), Bürgerkunde der Republik Österreich (1932), Österreichische Bürgerkunde (1936).

2 Mein Vaterland, mein Österreich (1898), Von Metternich bis Thun: 50 Jahre Österreich! (1899).

3 Unser Seelenleben im Völkerkriege: Ethische Betrachtungen (1915), Schicksal und Zukunft unserer Jugend (1931).

4 Tage der Wonne. Ägypten - Palästinareise (1931). 
die oben erwähnten Übersetzungen dar. ${ }^{1}$ Trotzdem hatte Herbatschek noch mehr Ergebnisse, und zwar in rein literarischer Hinsicht, vorzuweisen. Es handelt sich um seine eigenen fiktionalen Texte, ${ }^{2}$ in denen die zeittypischen Trends, insbesondere die Stilrichtungen der Moderne - obzwar mit ungefähr zwanzigjährigem Abstand, rezipiert wurden. Nicht zu verkennen sind dabei insbesondere die Anleihen bei den Wiener Modernisten, etwa die Spuren der Décadence und des Impressionismus. Herbatschek schrieb vorwiegend Prosa, darunter sind zwei Romane (Ist die Liebe tot? und F. G. A. Romantisches Hinterland) und Erzählungen. Er verfasste auch ein naturalistisch geprägtes Drama (Im Ausgedinge). Die naturalistische Schwärmerei ist als eine Art Vorstufe zur Moderne in der Ontogenese des Autors zu verstehen. Selbständige Lyriksammlungen konnten nicht entdeckt werden.

Obwohl sich Herbatschek (z. B. mit dem Roman Ist die Liebe tot?) zum großen Teil als raumgebundener Schriftsteller profilierte, sprengte er in einigen Werken (beispielsweise im analysierten Novellenband Skizzenbuch) völlig die eng gefassten Grenzen eines festgefügten geografisch-kulturellen und historisch-kulturellen Raums zugunsten grenzüberschreitender, zeit- und raumloser Themen. Und darin zeigt sich, neben der Heterogenität, auch der transitorische Charakter von Herbatscheks Prosa. Zur thematischen Struktur des Gesamtwerks lässt sich noch Folgendes sagen: Herbatschek verfolgte zwei prinzipielle thematische Linien - den Krieg und das Zusammenleben der Nationen. Der gemeinsame Nenner beider Linien ist die Bemühung um eine ethische Fundierung der Staats- und Gesellschaftsordnung. In der Erzählung Der Gottsfopper, die sich im Ersten Weltkrieg abspielt, überlebt ein österreichischer Soldat, hier ein Sprachrohr Herbatscheks, den Angriff der russischen Truppen. Nach der Rettung muss er einsehen, dass er als moralisierender Idealist und Individualist mit seinen existentiellen Erfahrungen nirgendwo hineinpasst. Er ist plötzlich ein Fremder unter den Eigenen, denn er entfremdete sich von seiner Umgebung, die sich keine Fragen bezüglich der Ethik des Lebens im Krieg stellt. Auch von hier führt sein spezifischer Weg in die Moderne.

Herbatschek widmete sich intensiv der Psychologie seiner Figuren. Hauptsächlich geht es um kleine, scheinbar unwichtige Menschen. Dementsprechend werden die Konflikte, welche die große Welt (etwa die Weltpolitik) bewegen, zunächst oder eben parallel in einer kleinen Welt ausgespielt. Der unpathetische Duktus

1 Neben dem oben erwähnten Buch Masaryks, Die Ideale der Humanität (1902), gab Herbatschek noch Machars autobiographisches Werk unter dem Titel Die Galeeren des Gymnasiums. Auslese aus „Bekenntnisse eines Literaten“ (1919) heraus, später noch einmal zusammen mit der Sammlung von Feuilletons Antike und Christentum. Herbatschek beteiligte sich auch an der Übersetzung und Herausgabe des Werkes Dichter Machar und Professor Masaryk im Kampfe gegen den Klerikalismus. In den 1930er Jahren übersetzte Herbatschek zwei Dramen des tschechischen Schriftstellers František Procházka (Justitia fundamentum und Pilgerfahrt der Ehe).

2 Im Ausgeding: Drama in 1 Akt (1909), Der Gottsfopper u. a. Kleine Erzählungen aus grosser Zeit (1915), Ist die Liebe tot? (1921), Skizzenbuch (1929), Justitia lacht! Eine Sammlung heiterer Dinge aus dem Rechts- und Anwaltsleben (1930), F. G. A. Romantisches Hinterland (1931). 
der Prosawerke Herbatscheks (mit Ausnahme des Dramas Im Ausgedinge, das ein klares naturalistisches Pathos ausstrahlt) entspringt einem quasi-dokumentarischen und pedantisch objektiven Stil, einem für die Darstellung der Entfremdung und Enttäuschung über die mangelnde Ethik durchaus passenden Medium. Die Figuren, allesamt Opfer des Krieges oder der modernen Zivilisation, suchen den Ausweg in praktischer Arbeit und Liebe.

Im Roman Ist die Liebe tot? bilanziert Herbatschek das Zusammenleben von Deutschen bzw. Juden und Tschechen, das er auf ein geistiges Niveau hebt, in einem interkulturellen Modellraum auf der Achse zwischen dem Zentrum (Wien) und der Provinz (die Modellstadt Altenburg). Das Österreichertum sieht er als Synthese des Slawischen und Germanischen.

\section{Skizzenbuch: Ein Jurist liest die Wiener Moderne}

Eine Ausnahmeposition unter den oben erwähnten Werken nimmt der Prosaband Skizzenbuch aus dem Jahr 1929 ein, in dem die für Herbatschek bis dahin unentbehrlichen regionalen, politischen oder rechtswissenschaftlichen Themen völlig ausgespart werden. Herbatschek begab sich mit und in diesem Buch auf ein Terrain, das ungefähr dreißig Jahre zuvor insbesondere die Autoren aus dem Umkreis des Jung-Wien für sich beansprucht hatten, nämlich in ein individuell-ästhetisches, elitäres, gott- und hierarchieloses Reich, das er nunmehr auch den Protagonisten seiner kürzeren Prosatexte gönnte.

Kann man aber wirklich Herbatscheks Schaffen im Kontext der Wiener Moderne behandeln? Die Nähe des Skizzenbuches zum Duktus repräsentativer Texte der Jung-Wiener, hauptsächlich in Bezug auf die Stil- und Ideenmerkmale, ist, wie auch später zu zeigen sein wird, unumstritten. Ernst zu nehmende Anzeichen für Herbatscheks Annäherung an die Zentralfiguren des Jung-Wien findet man jedoch - vor dem Erscheinen dieses Buches - eher im außertextuellen Bereich, nämlich in seinem Engagement für die Zeitung Die Zeit. Zu den prominenten Mitarbeitern der Tageszeitung Die Zeit (1902-1919) gehörten nämlich unter anderem die namhaften Repräsentanten der Wiener Moderne, Hugo von Hofmannsthal und Felix Salten, aber auch Tomáš Garrigue Masaryk, der Lieblingstscheche Herbatscheks. Die parallel erscheinende Wochenschrift Die Zeit (1894-1904) wurde von Hermann Bahr mitgegründet.

Einen wichtigen Anhaltspunkt für die Verwandtschaft mit den Wienern bietet die Form des Skizzenbuches. Die Skizze - kurzer, formal und stofflich bewusst nicht ausgeformter Prosatext - kam als literarische Ausdrucksform überraschenderweise bereits in Herbatscheks Der Gottsfopper zum Einsatz. Die in der Prosasammlung Skizzenbuch enthaltenen Skizzen, welche nun getreu der Tradition des Impressionismus überwiegend Eindrücke und Empfindungen eines Betrachters, weniger eine konkrete Handlung aufweisen, wurden dreiunddreißig Jahre nach dem 
Erscheinen des prototypischen impressionistischen Skizzenbandes Peter Altenbergs Wie ich es sehe (1896) herausgegeben. Eine Antwort auf die Frage zu finden, was für eine literarische Bedeutung dieses anachronistische Vorgehen haben könnte, gehört, neben der Eruierung des literarischen Beitrags des Werks, zu den Zielen der vorliegenden Analyse.

In Skizzenbuch lässt Herbatschek meistens einen wortgewandten und geschliffenen personalen Erzähler agieren, der die erlebte Rede und den inneren Monolog verwendet und der sich nur stellenweise um einen abgehackten, kurzatmigen Rhythmus bemüht:

„Bloß das Magische in den Blicken seiner Mutter schien ihm zu deuten, was er seine Jugend hindurch als Rätsel seines Charakters ergründen wollte ... Ja, den Weg würde er sicher nicht verfehlen: von Martigny über Orsières, geradeaus, dann rechter Hand durch ein Wäldchen, immer den Gipfel des St. Bernard vor Augen, bis Champex. Wie oft hatte die Mutter ihm die Gegend beschrieben? Jedes Dorf, jeder Pfad war ihr vertraut. Hatte sich seit jenem herrlichen Fest der Narzissen viel geändert? War das Leben weitergerollt oder die Welt stillegestanden?“ (Herbatschek 1929: 5)

Eine Antwort auf die letzte Frage könnte heißen: Das Leben ist weitergerollt und jetzt muss die Welt eine Weile stillstehen. In Narzissenfest wechseln Szenenfolgen mit Momentaufnahmen, wobei das an Impressionen reiche Leben der Figuren mit seinen unaufhaltsam dahinfließenden Augenblicken die eigentliche Dynamik des Erzählstoffes darstellt. Aufgrund der Beobachtung der inneren Wirklichkeit und der quälenden Selbsterforschung, ausgehend von beiläufigen Begegnungen und kurzen Dialogen, werden poetische Psychogramme der einzelnen Figuren à la Fin de siècle erstellt. Der Autor enthält sich - wie aber später zu zeigen sein wird, nur scheinbar - jeglicher Wertungen und Urteile, was er beispielsweise im besagten Werk Der Gottsfopper gerne und offen, z. B. in Bezug auf den Krieg, tut.

Herbatschek ändert in Skizzenbuch seine poetische Strategie, indem er sich auf die elementaren Entitäten wie Leben, Liebe und Tod konzentriert. Die damit kontrastierende Oberfächlichkeit der Darstellung hängt eng mit der übermäßigen Rolle der sinnlichen Wahrnehmungen zusammen, welche jeder Skizze ein dezidiert impressionistisches Gepräge geben. Laut Gotthart Wunberg (2001: 99) ist der Impressionismus ,...ein Schaltpunkt, an dem das im Naturalismus ausgebildete Verfahren [...] endgültig in die Selbständigkeit seiner Modernität entlassen wird." Herbatscheks Gesamtwerk an sich bestätigt diese Feststellung, denn man kann zweifelsohne in seinem dramatischen Einzelwerk Im Ausgeding (1909) ein naturalistisches Vorspiel zur Moderne und in Skizzenbuch dessen modernistische Fortsetzung erblicken. Der Impressionismus, der sich primär auf die Flüchtigkeit menschlichen Erlebens stützt, lässt - und dies gilt auch für Herbatscheks neue, in Skizzenbuch erprobte literarische Formensprache - die Phänomene der Außen- und 
der Innenwelt als Augenblickserscheinungen aufblitzen, in denen die Nähe und die Ferne, die Gegenwart und die Vergangenheit zusammenfallen.

Auch ein weiterer Schlüsselbegriff des Impressionismus repräsentiert die Momentaneität der ästhetischen Erfahrung, nämlich die Stimmung, die durch die Nerven, genauer gesagt durch die Reizmittel wie Gerüche, Farben, Geschmack, Klänge, erzeugt wird. Der Impressionismus ist laut Johnston (1980: 195) „die Auflösung jeglicher höheren Welt in einem Strom von Empfindungen." Johnstons These stützt sich auf den Empiriokritizismus Ernst Machs, der die Psyche als eine Entität, die aus isolierten Abfolgen von Ereignissen besteht, betrachtet; das Ich ist demnach als Einheit nicht mehr zu retten. Die impressionistische Betrachtungsweise wird häufig - auch in Skizzenbuch - mit einer stilisierten, zur Schau gestellten dekadenten Haltung kombiniert. In der Décadence erblickt Kafitz (2004) eine der Hauptrichtungen der Moderne, welche mannigfaltige, mit pathologischen Konnotationen besetzte Verfalls- und Niedergangsphänomene der Gesellschaft kurz vor der Jahrhundertwende repräsentiert.

Auch Herbatscheks Skizzen spiegeln diverse Verfallsphänomene wider, sie sprengen aber völlig den Zeitrahmen und wurzeln im Zeitlosen. In der ersten und in vieler Hinsicht tonangebenden Skizze, Narzissenfest, inszeniert Herbatschek die Genfer Seelandschaft als Handlungsort mit einem weltmännisch-internationalen Flair. Narzissenfest spielt sich zum Teil in einem noblen Berghotel, allem Anschein nach in Montreux, ab. Das Narzissenfest gehörte im Zeitraum 1897-1957 zu den Ereignissen des Jahres in Montreux - internationale Orchester traten dort auf, Umzüge mit den von Blumen dekorierten Wagen gingen durch die Stadt. Der Hauptprotagonist der Skizze, Konrad Sporck, will sich an einer ähnlichen Blumenfeier, die in der Nähe des Hotels abgehalten werden soll, beteiligen. Konrad, der sich als Ästhet und Solitär aus dem Geschlecht der Wiener Narzissen geriert, wartet nämlich auf ein Ereignis, das die klaffende Leere in seinem bisherigen Leben füllen und eine Wende durch Erkenntnis herbeiführen würde.

Narzissenfest liest sich wie ein Ausschnitt aus dem Leben Erwins, des viel bekannteren Propheten des Narzissmus und der Hauptgestalt der für die Moderne paradigmatischen Erzählung Der Garten der Erkenntnis von Leopold Andrian (1895). Dafür zeugen nicht nur die starke Mutterbindung Konrads und das immer wieder angedeutete geheimnisvolle Erbe seines Vaters, sondern auch das Fremdheitsgefühl und die hartnäckige Suche nach der Erkenntnis der Zusammenhänge der modernen Welt: „[...] moderne Menschen müssen die Wahrheit haben, sonst ist ihr ganzes Leben nicht nur Lüge von draußen, sondern auch Selbstbetrug [...]" (Herbatschek 1929: 13).

Die Handlung ist auf die Wiedergabe von anscheinend belanglosen Gesprächen und Erlebnissen reduziert. Der Text verfügt über ein Arsenal von Stimmungen, Duft-, Licht- und Farbwahrnehmungen. Die Außenwelt zerfällt in impressionistischer Manier, sie manifestiert sich spontan durch augenblicksartige Erscheinungen, von 
denen der Erzähler berichtet, wie etwa von „[...] dem unsagbar schönen Nachtkorso, den zahllosen Menschen, Fackeln, Lichtern und Raketen, [...] halben Sätzen und verstreuten Andeutungen, die wie farbige Perlen tröpfelten [...]" (Herbatschek 1929: 6). Viele Komponenten der Handlung sind aber reine Abstraktionen Erinnerungen an frühere Erlebnisse oder deren Reflexionen, also das, was im Geist existiert, nicht das, was in dem Augenblick den Erscheinungen inhärent wäre.

Das verwendete Handlungsmuster ist auf den ersten Blick banal. Auf der Darstellungsebene geht es um den Weg des Hauptprotagonisten zur Blumenfeier, welcher auf der symbolischen Ebene als die radikale Versenkung des Individuums ins eigene Ich und in die nicht besonders weit entfernte Vergangenheit zu verstehen ist. Dazu trägt auch der spezifische Handlungsraum bei, die Genfer Seelandschaft, die eine moderne Transformation der ursprünglich regionalen, politisch oder national aufgeladenen Räume aus den früheren Werken Herbatscheks zu sein scheint.

Eine derart transformierte Landschaft ermöglicht eine effektivere Sondierung in die Subjektivität des modernen Menschen, in seine Neurotizität, Sensibilität und Isolation (hierzu siehe Kafitz 2004: 223 f.). Dementsprechend werden in diesem neu konstruierten Locus poeticus die ursprüngliche, für Herbatscheks Werke typische Dynamik in eine ästhetisierte Statik und die konkreten Handlungen in eine Reihe von Ritualen transformiert (z. B. die Fahrt auf der Lagune, die Wahl der Königin des Festes etc.) - ein ideales Arrangement für die Entfaltung des Ästhetentums und für die übersteigerte Ichbezogenheit Konrads. Das Narzissenfest avanciert somit einerseits zur Initiation Konrads in existentieller und noetischer Hinsicht und andererseits zur Feier des modernen Lebens. Für die dekadente Ausrichtung des Festes sorgen dann die traditionellen Topoi und Attribute der Décadence wie Nacht, Lagune, hell erleuchtete Boote, Fackeln, Lampions, Garben der Narzisse, müde Menschen, Musik, feierliche Umzüge und schließlich der Tod.

Der Verlauf des Narzissenfestes ist durch einen tragischen Vorfall gekennzeichnet - durch den Tod einer unbekannten Dame, die sich beim abschließenden Zeremoniell in den See stürzt. Aus den Anspielungen des Erzählers kann man darauf schließen, dass es sich um die Mutter Konrads handelt, die kurz nach der Ankunft in Montreux freiwillig aus dem Leben scheidet. Konrads Vater, ein Lebemann und Lebenskünstler, lernte vor vielen Jahren seine Frau gerade während des Narzissenfestes kennen. Offensichtlich unterhielt er aber auch weiterhin Beziehungen zu anderen Frauen. Allem Anschein nach hatte er vor vielen Jahren mit einer von ihnen das Mädchen namens Thekla gezeugt, das nun - samt ihrer Mutter -Konrad im Berghotel rein zufällig begegnet, und zwar unmittelbar vor dem Anfang des Festes. Thekla ist, wie man vielleicht erwarten könnte, keine schwächliche Femme fragile aus dem Lehrbuch der Wiener Moderne. Sie weist auch keine stilisierte Laszivität oder Verlogenheit auf, eine inzestuöse Beziehung mit Konrad kommt ebenfalls nicht zustande. Thekla stellt eher ein Surrogat Gottes dar - wie übrigens ihr Name verrät (vom altgriechischen Namen Theokleia - die von Gott Gerufene). Dementsprechend mahnt sie Konrad 
zur Reflexion und Erkenntnis. Theklas Mutter, die ehemalige Geliebte von Konrads Vater, ist eine Femme fatale, nur insofern die Begegnung mit ihr den Selbstmord ihrer lebenslangen Rivalin, der Mutter Konrads, herbeiführt.

Hinter dem impressionistischen Arrangement der Skizze verbirgt sich eine feinsinnig durchdachte Rollenkonstellation: der gleichgültige Ästhet und autistische Moralprediger Konrad, Thekla als verkörperte Provokation und Warnzeichen eines nicht mehr existierenden Gottes, Theklas Mutter als Schicksalsfrau, Konrads Vater als Prototyp des modernen Menschen ohne moralischen Code, Konrads Mutter als tragische Opferfigur. Herbatschek zeigt dabei eine beinahe juristische Zurückhaltung und Umsicht, er bewegt sich um jeden Preis im Rahmen des gesetzlich Erlaubten und vermeidet Exzentrizitäten. Insgesamt lässt sich in Narzissenfest beobachten, dass die in der Moderne enttabuisierten Themen zwar aufgeworfen, aber sofort uminterpretiert oder verschleiert werden. Bis zum Ende werden einige Geheimnisse gewahrt, beispielsweise das Geheimnis des Todes von Konrads Vater, für weitere sorgen die unzähligen Leerstellen in der Handlungsstruktur. Die rückwärtsgewandte, in Impressionen und symbolische Gesten zerfallende Auseinandersetzung mit der Vergangenheit gibt schließlich das Geheimnis von Konrads Leben preis - bis zum Narzissenfest hielt ihn nämlich „eine Kraft zurück, deren Quell ihm nicht bewußt war" (Herbatschek 1929: 5). Die erkannte Wahrheit über seine Familienverhältnisse, über seinen leichtsinnigen Vater, die Folgen seines verantwortungslosen Handelns und der daraus resultierende Tod seiner Mutter generieren eine klare Botschaft: Für frivole Abenteuer, auch für diejenigen der Väter, wird man früher oder später bezahlen. Auch in der modernen Welt, oder vielleicht gerade in der modernen Welt, muss man der Freiheit Grenzen setzen, und zwar durch die aufklärerischen Werte Vernunft und Moral. Das Leben, das sich als Fest, Rausch und Schönheit gestaltet, ist im Endergebnis lebensgefährlich. In das komplizierte ästhetische und dekadente Arrangement des Narzissenfestes fand also - mit einer beinahe didaktischen Raffinesse - eine moralisierende Mahnung Eingang. Die ästhetische Moderne wird in Narzissenfest postuliert und zugleich mit ihren eigenen Mitteln überwunden. Das unrettbare Machsche Ich bemüht sich doch um eine Rettung.

Auch weitere Skizzen des Prosabandes bieten mannigfaltige Facetten der ästhetischen Moderne - mit Modifizierungen. Das in Narzissenfest erörterte Thema Suizid wird in der Skizze D'Ferdinandsbruck' $n$ wieder aufgegriffen. Der für viele Texte der Wiener Moderne typischen, psychologisierenden Perspektive geht hier noch ein historischer Exkurs voraus. Es werden drei Ereignisse aus der Geschichte der Wiener Ferdinandsbrücke festgehalten und durch die Thematik des Todes verbunden: die Legende von ihrer Gründung im 14. Jahrhundert, das turbulente Geschehen im Revolutionsjahr 1848 und das Ende des Ersten Weltkriegs. Die Brücke symbolisiert jeweils die durchlässige Grenze zwischen Leben und Tod - in welcher Form und Epoche auch immer er auftritt. 
Die Titelheldin der Skizze Nora Hofmann ist eine geheimnisvolle, der Spionage verdächtigte Frau. In der Haft erträgt Nora stoisch alle Verhöre, die impressionistisch nur auf Empfindungen reduziert werden. Die als Häftling in einem Kloster untergebrachte Nora zieht die Aufmerksamkeit der Nonne Placida auf sich. Die Skizze kreist ähnlich wie Narzissenfest um die Themen Liebe und Freiheit. Die lesbische Liebe zwischen Placida und Nora steht allerdings nicht im Mittelpunkt des Geschehens, wie man erwarten würde. Nora strebt Freiheit an und will aus dem Gefängnis fliehen, Placida sucht Liebe und hilft Nora aufopferungsvoll bei ihrer Flucht aus dem Kloster. Die grundsätzliche Frage dieser Skizze lautet: Welchen Stellenwert nimmt im Leben des modernen Menschen die Freiheit ein und was alles ist er bereit für die Freiheit zu opfern?

Der Kompagnon (Ein Romanfragment) ist eine Sondierung der Psyche eines Heranwachsenden, Wilhelm Viktors, der isoliert lebt und sich völlig seinen sexuellen Fantasien hingibt. Der Dachboden, auf dem er sich oft bewegt, ist ein Abbild für seine durch Triebe und Schuld zerrissene Seele. Hier trifft er auch die Untermieterin und Geliebte des örtlichen Pfarrers, die er vergewaltigt. Diesen Prototyp der Frau sucht Wilhelm anschließend in jeder anderen Frau. Die geistige Verwandtschaft der Skizze mit Robert Musils Törleß-Roman ist hier kaum zu verkennen.

Im Unterschied zu dem intellektuell veranlagten Konrad Sporck und dem sadistisch orientierten Wilhelm Viktor ist der Hauptprotagonist der mystisch anmutenden Skizze Herzbub ein Symbol für die ästhetisch fundierte Maskulinität und zugleich für das männlich regierte Abendland. Die Kunst, hier durch eine Statue repräsentiert, steht wiederum für das weibliche Prinzip. Die Kunst und der rauschhafte Genuss des Lebens stellen in Herbatscheks Auffassung ein gefährliches und regressives Element dar, ähnlich wie in Narzissenfest. Im dramatischen Fragment Nach der Scheidung, das ein Gespräch von zwei jungen Frauen festhält, bietet Herbatschek eine andere Sicht auf die Maskulinität, nämlich Männlichkeit als Last trotz ihrer bewegenden Kraft.

Die in die chinesische Vergangenheit verlegte Skizze Liebet euch erzeugt eine tragikomische Illusion von der humanen Staatsmacht, die als moralische Utopie präsentiert wird. Nu-cha-min, ein in der chinesischen Provinz lebender Gutspächter, setzt sich für die Befreiung Ku-hus, eines Dissidenten, ein, der von der Staatsmacht ungerecht beschuldigt wurde. Nu-cha-min stirbt bei einem Anschlag, der das Staatsoberhaupt paradoxerweise zu keinen weiteren Repressionen, sondern zur Umkehr und humanen Lösungen veranlasst.

In der ästhetisch und dekadent gefärbten Skizze Das Sujet werden die Qualen eines jungen Autors geschildert, der die Unmöglichkeit, ein neues Sujet für seine Texte zu erfinden, thematisiert. Die Fiktionalität und das Schreiben steigen hier zum Symbol für das Leben selbst auf. Alle Lebensentwürfe scheinen dekadent - alt, unbrauchbar, überwunden, ja tot - zu sein. Die ganze Skizze kippt schließlich ins Komische. 


\section{Fazit}

Die Analyse des vorliegenden Prosabandes Skizzenbuch von Heinrich Herbatschek und die Erkenntnisse über den gesellschaftlichen und historischen Kontext von Herbatscheks literarischer Tätigkeit ergaben Befunde, die sich in den folgenden Thesen zusammenfassen lassen. Die deutsche Literatur aus der Mährischen Walachei verdient eine seriöse und komplexe Erforschung, denn sie bietet bemerkenswerte kultur- und literaturhistorische Phänomene, welche einerseits Beweise für die Rezeption der ästhetischen Moderne seitens der marginalen und regionalen Autoren, andererseits Belege für die Sonder-, Um- und Abwege der Moderne liefert. Es wäre wünschenswert, das Werk Heinrich Herbatscheks (1877-1956) im Kontext der ästhetischen Moderne zu erforschen. Aus dieser Perspektive wird die Moderne als eine relevante Schnittstelle erkennbar, die den sich in der Literatur und Gesellschaft vollziehenden Paradigmenwechsel anzeigt. Heinrich Herbatscheks Skizzenbuch (1929) ist ein weithin unrezipiert gebliebener spätmodernistischer Text, der sich impressionistischer und dekadenter Formensprache bedient. Das modernistische Arrangement geht hier eine ambivalente Symbiose mit traditionellen, humanistischaufklärerischen Wertvorstellungen ein. Herbatschek stellt sein Werk in den Dienst der Moderne, sein Schaffen erscheint aber weiterhin kohärent.

\section{Literaturverzeichnis}

\section{Primärliteratur:}

Altenberg, Peter (1896): Wie ich es sehe. Berlin, S. Fischer.

Andrian, Leopold (1895): Der Garten der Erkenntnis. Berlin: S. Fischer.

Herbatschek, Heinrich (1909): Im Ausgeding. Drama in 1 Akt. Leipzig [u.a.], Reform-Verlag. Herbatschek, Heinrich (1913): Aus dem Bildersaal eines verkannten Kulturvolkes. Wien, Kommissionsverlag des Deutsch-czechischen Komitees.

Herbatschek, Heinrich (1915): Der Gottsfopper u. a. Kleine Erzählungen aus grosser Zeit. Wien, Perles.

Herbatschek, Heinrich (1921): Ist die Liebe tot? Wien, Gesellschaftsverlag.

Herbatschek, Heinrich (1929): Skizzenbuch. Wien, Frisch.

Herbatschek, Heinrich (1930): Justitia lacht! Eine Sammlung heiterer Dinge aus dem Rechtsund Anwaltsleben. Wien, Selbstverlag.

Herbatschek, Heinrich (1931): F. G. A. Romantisches Hinterland. Wien, Dr.-HerbatschekVerlag.

Masaryk, Thomas Garrigue (1902): Die Ideale der Humanität. Wien, Konegen.

\section{Sekundärliteratur:}

Bartoš, Josef / Schulz, Jindřich / Trapl, Miloš (1980): Historický mistopis Moravy a Slezska $v$ letech 1848-1960, sv. VII. Ostrava, Profil.

Blumesberger, Susanne / Doppelhofer, Michael / Mauthe, Gabriele (Red.) (2002): Handbuch österreichischer Autorinnen und Autoren jüdischer Herkunft. 18. bis 20. Jahrhundert. Hrsg. von der Österreichischen Nationalbibliothek. München, Saur. 
Brouček, Stanislav / Jeřábek, Richard (eds.) (2007): Lidová kultura. Národopisná encyklopedie Čech, Moravy a Slezska, sv. 3. Praha, Mladá fronta.

Hájek, Tomáš (2009): Heinrich Herbatschek als Herausgeber der Zeitschrift „Der Nachbar“. Diplomová práce. Filozofická fakulta Masarykovy univerzity.

Johnston, William M. (1980): Österreichische Kultur-und Geistesgeschichte. Gesellschaft und Ideen im Donauraum 1848 bis 1938. Wien (u.a.), Böhlau.

Kafitz, Dieter (2004): Décadence in Deutschland. Studien zu einem versunkenen Diskurs der 90er Jahre des 19. Jahrhunderts. Heidelberg, Universitätsverlag Winter.

Krappmann, Jörg (2012): Komplexität, Schlichtheit und Abstraktion in der regionalen Literaturforschung. In: Horňáček, Milan / Voda Eschgfäller, Sabine (Hg.): Regionalforschung zur Literatur der Moderne. Olomouc, Univerzita Palackého v Olomouci. S. 23-39.

Krappmann, Jörg (2013): Allerhand Übergänge. Interkulturelle Analysen der regionalen Literatur in Böhmen und Mähren sowie der deutschen Literatur in Prag (1890-1918). Bielefeld, Transcript.

Weinberg, Manfred (2014): Die Grenzen der Interkulturalität. In: Fiala-Fürst, Ingeborg (Hg.): Prager deutsche, deutschböhmische und deutschmährische Literatur. Eine Neubestimmung.

Olomouc, Univerzita Palackého v Olomouci. S. 7-32.

Wunberg, Gotthart (2001): Jahrhundertwende. Studien zur Literatur der Moderne. Tübingen, Gunther Narr.

\section{Internetquellen:}

Dr. jur. Heinrich Herbatschek. In: Geni. https://www.geni.com/people/Dr-Jur-Heinrich-He rbatschek/6000000011645007401\#/tab/overview

Libor Marek

Univerzita Tomáše Bati ve Zlíně

marek@utb.cz 